Research

\title{
Impact of an automated Internet-based cognitive behavioral therapy program on suicide thinking and risk among United States rural adults
}

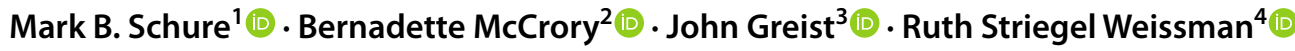

Received: 12 October 2021 / Accepted: 7 February 2022

Published online: 03 March 2022

(c) The Author(s) 2022 OPEN

\begin{abstract}
Cognitive behavior therapy (CBT) is an evidence-based psychotherapy for mental disorders, including depression. Internet-based CBT (iCBT) programs increasingly are showing similar impact to clinician-delivered CBT. We assessed the impact of Thrive, a fully-automated iCBT depression treatment program on suicidal thinking. Participants were randomly assigned to the intervention (INT) group $(n=218)$ or a waitlist control group (WLC, $n=230)$. Intent-to-treat analyses tested for group differences at 8-weeks in suicidal thinking (CHRT-SR 3 subscale, primary outcome), and secondary outcomes including depression symptoms (PHQ-9), anxiety symptoms (GADS-7), work and social adjustment (WSAS), and resilience (CD-RISC-10). Using self-reports, participants were evaluated at baseline, 4 and 8 weeks for each outcome. Thrive program adherence $(n=218)$ was assessed by number of lessons completed. Although not statistically significant, the INT group was $38.7 \%$ less likely than the control group to present with suicidal thinking at 8 weeks (odds ratio $0.61, p=0.10$ ). Comparison of 8-week depression symptom slopes showed statistically significant effects favoring the INT group (WLC $=-3.04$ vs Thrive $=-4.32, p=0.007)(d=0.08)$; no other significant group differences were observed. Lessons completed were significantly related to lower PHQ-9 $(p=0.026)$ and GAD-7 scores $(p<0.01)$. Study results are consistent with a previous study showing nonsignificant effect of an automated iCBT program for reducing suicidal thinking, but a significant positive impact on depression symptoms among rural US adults. Future studies should test whether strategies for boosting lesson completion are successful in enhancing the efficacy of Thrive to reduce suicide risk.

Trial Registration: National Institutes of Health Trial ID: NCT03595254.
\end{abstract}

Keywords Internet-based cognitive behavior therapy · Suicide risk - Depressive symptoms · Rural populations · Effectiveness trial

\section{Introduction}

Epidemiologic studies have consistently found greater suicide prevalence and trends of increasing suicide rates among US rural populations compared to urban populations [1-9]. Data from 2008 to 2018 show age-adjusted suicide rates growing faster in rural areas compared to urban areas with an increase of $48 \%$ compared to $34 \%$ [8]. Hence, there is a need to address risk factors for rural US adults.

$\triangle$ Mark B. Schure, mark.schure@montana.edu | ${ }^{1}$ Department of Health and Human Development, Montana State University, 305 Herrick Hall, Bozeman, MT 59717, USA. ${ }^{2}$ Department of Mechanical and Industrial Engineering, Montana State University, Bozeman, MT 59717, USA. ${ }^{3}$ Department of Psychiatry, School of Medicine and Public Health, University of Wisconsin, 6001 Research Park Blvd, Madison, WI 53719, USA. ${ }^{4}$ Department of Psychology, Wesleyan University, 207 High Street, Middletown, CT 06459, USA.

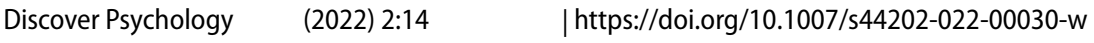


Research has identified a number of factors placing rural communities at greater suicide risk, including greater geographic or social isolation, economic strain, access to lethal means such as guns, and long distances to and/or limited availability of mental health treatment resources compared to urban or suburban communities [10]. In the US, Western mountain states have been labeled the "suicide belt", with higher suicide rates compared to other states [11]. The vast majority of communities in these states are rural and have significantly greater barriers to accessing mental health care, including stigma, cost, and inability to schedule appointments. For example, in Montana, more than $50 \%$ of counties do not have a psychologist; over $65 \%$ do not have a psychiatrist [12]. Other universal barriers including stigma, cost, and inability to schedule appointments are also present [13]. Yet, the need for care is comparably greater in Montana, given the state's higher depression prevalence than the national rate (24.1\% vs $19.7 \%)$ [14] and its long-term consistent top three ranking of states with the highest suicide rates in the nation [15].

Given greater barriers to care, novel solutions are needed to address suicide risk in rural communities. One recommendation is to disseminate Internet-based psychoeducation programs [16]. Internet-based programs that teach Cognitive Behavior Therapy (iCBT) skills have emerged as evidence-based solutions to address depression and anxiety symptoms [17]. Fully-automated versions of iCBT programs (those not requiring human support) have the additional advantage of partially addressing the enduring shortage of mental health care providers [18].

Currently, most iCBT studies have been conducted outside of the US [19]. This study is one of two randomized clinical trials (RCT) evaluating a culturally adapted iCBT program called "Thrive" with rural US adults [20]. The Thrive program draws upon evidence-based CBT principles designed to reduce depression symptoms. The first Thrive trial with 343 adults demonstrated at 8-weeks significant and clinically meaningful reductions in depression and anxiety symptoms (effect sizes $d=0.63$ and 0.47 , respectively) in the intervention (INT) group compared to the waitlist control (WLC) group [21]. Furthermore, longitudinal analyses showed that these improvements were sustained for 12 months [22]. The present study builds upon and extends this earlier work with the primary aim to assess the impact of the Thrive intervention on suicidal thinking as the primary outcome measure by measuring suicidal thinking (rather than depressive symptoms) as the primary outcome. A second aim was to assess the relationship between program adherence and outcomes. Based on our prior research, we hypothesized that participants in the INT group would show significantly great reductions in suicide risk (primary outcome) and in depression and anxiety symptoms (secondary outcomes)."

\section{Methods}

\subsection{Trial design}

We used a randomized controlled trial (RCT) design, randomly assigning participants to immediate Thrive program access (INT group) vs 8-week delayed access to Thrive (WLC group). Potential participants were provided with a link to the study website, consented electronically, and presented with the same assessment battery at study entry (baseline), 4- and 8-weeks. Study eligibility criteria included being at least age 18 years, Montana residency, a score of $\geq 5$ on the Patient Health Questionnaire (PHQ)-9 [23], and having access to a device (computer, tablet, or smart phone) with broadband Internet. Compared to the first trial, the current study provided more modest incentives for research participation ( $\$ 10$ vs $\$ 25$ with the first trial) for each completed follow-up assessments. The study protocol was approved by the Institutional Review Board of Montana State University. The trial was registered at nihtrials. gov: NCT03595254.

\subsection{Participants}

Participants included adults aged 18 years and over (mean age $=42.7$ ) with residence in the state of Montana. Over $80 \%$ were female and nearly $90 \%$ were White (see Table 1 ). Nearly three quarters resided in designated rural parts of the state. Over $50 \%$ of participants reported having a bachelor's or higher degree; nearly $60 \%$ reported receiving some form of mental health treatment at baseline. 


\subsection{Procedure}

Participants for the study were recruited between November 2018 and April 2020 using presentations to health care providers, flyers, posters, social media advertisements, and state Extension agents. Of 1102 individuals assessed for eligibility, 729 were deemed eligible and consented to participate. A simple computer auto-generated program was used to randomize 366 INT group participants and 363 WLC group participants. The analytic sample was further reduced by eliminating those who either: (1) were deemed fake participants (we refer readers to the first Thrive RCT publication [21] for details concerning identification of fake participants), (2) entered invalid email addresses, (3) reflected duplicate participation or entered an invalid date of birth, or (4) did not complete the suicide risk baseline assessment. The final analytic sample included 218 INT group and 230 WLC group participants (see Fig. 1). All participants were told to keep receiving the care that they were currently using.

\subsection{Intervention description}

Thrive is a fully automated intervention for depression based on CBT principles and techniques, including cognitive restructuring, behavioral activation, and social skills training [24]. These CBT themes are captured in each module (Constructive Thinking, Rewarding Activities, and Assertive Communication) with each module containing 10 lessons and suggested exercises for users to practice off-line as homework pertinent to their own self-identified goals. Thrive uses videos, interactive tools, and sophisticated algorithms that dynamically adjust the individual's use of the intervention, including a suite of 320 videos that explain CBT concepts, demonstrate skills, and present case histories of individuals who used CBT skills to improve depression symptoms. The program also features periodic PHQ-9 self-assessments, followed

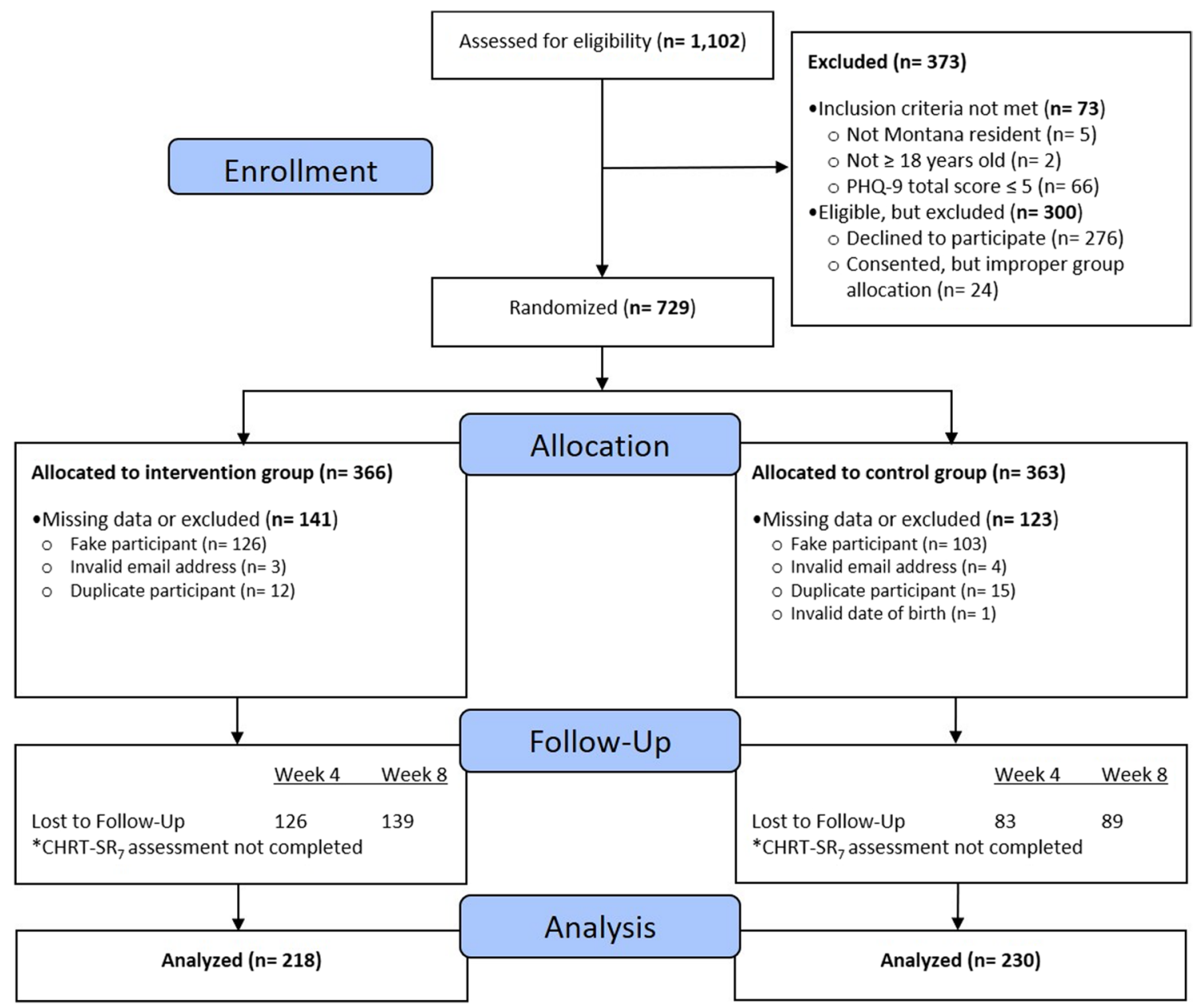

Fig. 1 Study consort flow chart. "Improper group allocation" occurred via an automated randomization glitch 
Fig. 2 Eight-week trend comparisons of suicidal thinking. Suicidal risk as measured using 3 items of the 7-item Concise Health Risk Tracking Self-Report [25, 26]. INT Group immediate Thrive intervention group; WLC Group waitlist control group

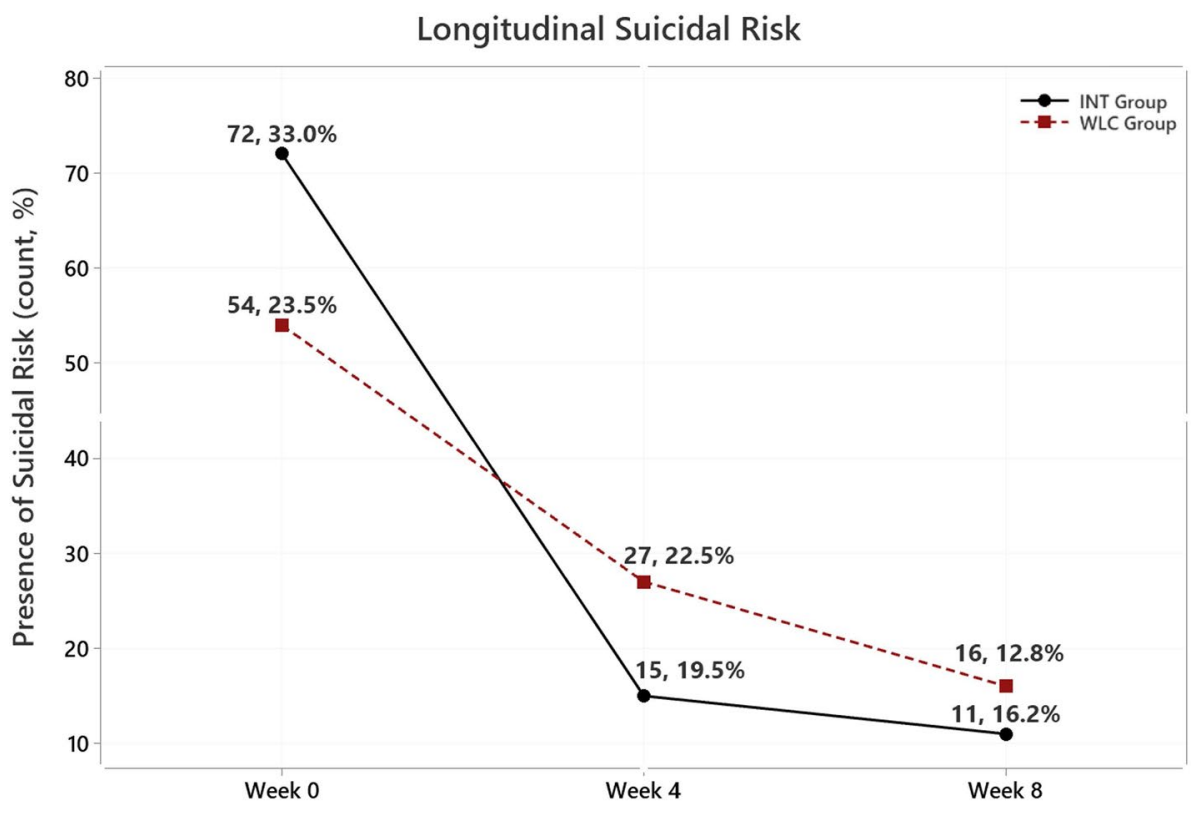

by tailored feedback based on the scores. Over a third of the demonstration and case history videos were replaced with new videos featuring rural characters, story lines, and settings; all other features of the Thrive program (i.e., didactic videos, interactive tools, and algorithms) were unchanged.

\subsection{Study instruments}

\subsubsection{Demographic variables}

Participant demographic information included age (years), gender (female vs male), race (White vs other), marital status (married/domestic relationship vs all other statuses), employment status (employed full-time/part-time/student vs unemployed/retired), educational attainment (some college without a degree, bachelor's degree, master's degree or higher), and rural classification (urban vs large rural/small rural/isolated). Participants' addresses (zip codes) were used to determine 10 rural-urban commuting area (RUCA) codes which classify US census tracts based on population density, urbanization, and daily community (USDA Economic Research Service, 2020). These codes were further collapsed into 4 codes: urban, large rural, small rural, and isolated. Participants also self-reported whether they were receiving any care or taking medication(s) for mental health treatment.

\subsubsection{Primary outcome measure}

The primary outcome measure was the 3-item Suicidal Thinking subscale $\left(\mathrm{CHRT}_{3}\right)$ of the 7-item Concise Health Risk Tracking Self-Report (score range: 3-15, higher scores indicate greater suicidal thinking in the past 2 weeks; Cronbach Alpha $=0.80)[25,26]$. The measure's three items were combined and dichotomized to form a binary outcome measure of suicidal thinking (agreement/disagreement). The three items are: "I have been having thoughts of killing myself", "I have thoughts about how I might kill myself," and "I have a plan to kill myself. An "agree" or "strongly agree" response on any of the three items indicated presence of suicidal thinking (coded as 1). Participants who self-reported neither agree nor disagree, disagree, or strongly disagree on all 3 items indicated an absence of suicidal thinking (coded as 0 ). 


\subsubsection{Secondary outcome measures}

Pre-specified secondary outcomes included: depression symptom severity, measured using the 9-item Patient Health Questionnaire (PHQ-9; score range: 0-27; higher scores indicate greater frequency of symptoms in the past 2 weeks) [23]; anxiety symptom severity, measured with the Generalized Anxiety Disorder Scale (GADS-7; score range: 0-21; higher scores indicate greater frequency of symptoms in the past 2 weeks) [27, 28]; daily functioning, using the Work and Social Adjustment Scale (WSAS; score range: 0-40; higher scores indicate a greater adverse impact of depression on daily functioning) [29]; and resilience, as measured by the Conner-Davidson Resilience Scale (CD-RISC-10; score range: 0-40; higher scores indicate greater resilience) [30].

\subsection{Data analyses}

The dichotomized primary outcome variable was modeled using a logistic regression model within a Generalized Estimating Equation (GEE) framework with repeated measures to compare the INT and WLC groups suicidal risk over time [31]. This model contained fixed-effects terms for treatment, time (baseline, week 4, week 8), treatment $\times$ time interaction, and the baseline mean suicidal thinking score as a covariate. For easier interpretation of the treatment effect, odds ratios were estimated as part of the binary logistic model. The change over 8 weeks in the for each continuous secondary outcome measures was compared between the INT and WLC groups using a linear mixed model analysis of repeated measures [21]. All models contained fixed-effects terms for treatment, time (baseline, week 4, week 8), treatment $\times$ time interaction, and the respective baseline mean score for each outcome (prior to the intervention) as a covariate. For better interpretation of the treatment effect, the least squares mean (adjusted treatment mean scores) were estimated as part of each mixed model. Rates of remission and relapse were assessed longitudinally for the INT group using the PHQ-9. Remission was defined as a treatment response in which an individual with mild, moderate, moderately severe, or severe depression at baseline (PHQ-9 scores $\geq 5$ ) achieved a subsequent PHQ-9 score lower than 5 at 4 weeks and/or 8 weeks. Relapse was defined as a PHQ-9 score $\geq 10$ for those who had achieved remission at 4 weeks.

Analyses of Thrive program adherence (INT group only) were completed by assessing the longitudinal change over time in the outcomes to assess the relationship with lessons completed in the program. Using separate logistic regression models for the primary outcome and linear mixed models with repeated measures for secondary outcomes, the adherence measure (lessons completed) was assessed at baseline, 4 weeks, and 8 weeks for each outcome [22]. All models contained fixed-effects terms for the lessons completed, time (baseline, week 4, week 8), and respective baseline outcome measure as a covariate. Statistical analyses were performed using SAS software (Version 9.4, SAS Institute, Inc., Cary, North Carolina). Maximum likelihood estimators allow efficient parameter estimation using only available data under an assumption of missing at random [32-34]. The level of significance was set at $a=0.05$ (two-tailed). Bonferroni adjustments were assessed to ensure the $95 \%$ confidence intervals for the point estimates of treatment group effects matched the significance levels in the corresponding test. A priori evaluable sample size for a statistical power of $80 \%$ was estimated (enrollment of $n=99$ per group).

\section{Results}

\subsection{Treatment outcomes}

Table 2 presents the longitudinal mean 8-week trends for the primary outcome. Suicidal ideation was reported by $28.1 \%$, $17.6 \%$, and $12.3 \%$ of participants at baseline, week 4 , and week 8 , respectively. The predicted odds of elevated suicidal risk $\left(\mathrm{CHRT}_{-} \mathrm{SR}_{3}\right)$ for the intervention group showed a steeper decline from baseline to week 8 than that of the control group but this difference failed to reach statistical significance; the INT group was $38 \%$ less likely than the WLC group to present with suicidal thinking following the entire 8-week follow-up period (see Fig. 2).

Table 3 presents the longitudinal mean 8-week trends for each secondary outcome. We found a statistically significant depression severity slope showing contrasts favoring the INT group. No significant between-group slopes were observed for the remaining secondary outcomes.

All INT group participants began the study with a PHQ-9 score of 5 or above $(n=218)$. A large majority $(77.1 \%)$ had moderate or greater depression symptom severity (i.e., PHQ-9 scores $\geq 10, n=168$ ). For those with follow-up data $(n=79)$, 
Table 1 Study sample baseline characteristics

\begin{tabular}{|c|c|c|c|c|c|c|}
\hline \multirow{3}{*}{$\begin{array}{l}\text { Study group } \\
\text { Characteristics } \\
\text { Age, mean (SD) }\end{array}$} & \multirow{2}{*}{\multicolumn{2}{|c|}{$\frac{\text { Total sample }}{\mathrm{n}=448}$}} & \multirow{2}{*}{\multicolumn{2}{|c|}{$\frac{\text { WLC group }}{n=230}$}} & \multirow{2}{*}{\multicolumn{2}{|c|}{$\frac{\text { INT group }}{n=218}$}} \\
\hline & & & & & & \\
\hline & \multirow{2}{*}{$\begin{array}{l}42.7 \\
n\end{array}$} & \multirow{2}{*}{$\frac{(13.7)}{(\%)}$} & \multirow{2}{*}{$\begin{array}{l}43.5 \\
n\end{array}$} & $(13.3)$ & 41.9 & $(14.1)$ \\
\hline & & & & (\%) & $\mathrm{n}$ & (\%) \\
\hline Baseline suicidal thinking ${ }^{a}$ & 126 & $(28.1)$ & 54 & $(23.5)$ & 72 & $(33.0)$ \\
\hline Female $\left(n^{*}=15\right)$ & 360 & $(83.1)$ & 183 & $(82.4)$ & 177 & $(83.9)$ \\
\hline \multicolumn{7}{|l|}{ Race $\left(n^{*}=18\right)$} \\
\hline White & 385 & $(89.5)$ & 199 & $(90.1)$ & 186 & $(89.0)$ \\
\hline All others ${ }^{b}$ & 45 & $(10.5)$ & 22 & $(10.0)$ & 23 & $(11.0)$ \\
\hline \multicolumn{7}{|l|}{ Marital status $\left(n^{*}=11\right)$} \\
\hline Married/domestic partnership & 237 & $(54.2)$ & 128 & $(56.6)$ & 109 & $(51.7)$ \\
\hline All other statuses & 200 & $(45.8)$ & 98 & $(43.4)$ & 102 & $(51.7)$ \\
\hline \multicolumn{7}{|l|}{ Employment status $\left(n^{*}=11\right)$} \\
\hline Employed full/part-time/student & 351 & $(80.3)$ & 185 & $(81.9)$ & 166 & $(78.7)$ \\
\hline Unemployed/retired & 86 & $(19.7)$ & 41 & $(18.1)$ & 45 & $(21.3)$ \\
\hline \multicolumn{7}{|l|}{ Education $\left(n^{*}=11\right)$} \\
\hline Associate degree, some college or less & 213 & $(48.7)$ & 98 & $(43.4)$ & 115 & $(54.5)$ \\
\hline Bachelor's degree & 141 & $(32.3)$ & 75 & $(33.2)$ & 66 & $(31.3)$ \\
\hline Master's degree or higher & 83 & $(19.0)$ & 53 & $(23.5)$ & 30 & $(14.2)$ \\
\hline Rural classification $^{c}$ & 328 & $(73.2)$ & 174 & $(75.7)$ & 154 & $(70.6)$ \\
\hline Receiving mental health treatment ${ }^{d}\left(n^{*}=11\right)$ & 260 & $(59.5)$ & 128 & $(56.6)$ & 132 & $(62.6)$ \\
\hline
\end{tabular}

WLC waitlist control; INT intervention; $n^{*}$ number missing

${ }^{a}$ Suicidal thinking (Items 5, 6 and 7) on the Concise Health Risk Tracking Scale-Self Report 7-Item (CHRT-SR $\left.{ }_{7}\right)$ was compared between the two groups (INT vs WLC) as agreement (presence) with suicidal risk (yes/no)

${ }^{b}$ Other race included Al/AN, Hispanic, Asian, Black/African American, Native Hawaiian/Other Pacific Islander, Some other race or origin

${ }^{\mathrm{C}}$ Defined using RUCA (rural urban commuting area) codes. Includes only rural residents (codes 2-4)

${ }^{\mathrm{d}}$ Receiving any care or taking medication(s) for mental health treatment

Table 2 Effect of Thrive intervention on $\mathrm{CHRT}^{-\mathrm{SR}_{7}}$ suicidal thinking at each timepoint and overall

\begin{tabular}{|c|c|c|c|c|c|c|c|c|c|c|c|c|}
\hline & \multicolumn{4}{|c|}{ Odds of suicidal risk at week 4} & \multicolumn{4}{|c|}{ Odds of suicidal risk at week 8} & \multicolumn{4}{|c|}{ Overall odds of suicidal risk ${ }^{d}$} \\
\hline & $\mathrm{OR}^{\mathrm{a}}$ & $95 \% \mathrm{Cl}^{\mathrm{C}}$ & Z-statistic & $\overline{p \text {-value }}$ & $\overline{O^{b}}$ & $95 \% \mathrm{Cl}^{\mathrm{C}}$ & Z-statistic & $\overline{p \text {-value }}$ & $\mathrm{OR}^{\mathrm{b}}$ & $95 \% \mathrm{Cl}^{\mathrm{C}}$ & Z-statistic & p-value \\
\hline INT group ${ }^{a}$ & 0.37 & $(0.12,1.16)$ & -1.70 & 0.09 & 0.48 & $(0.14,1.66)$ & -1.16 & 0.25 & 0.61 & $(0.31,1.22)$ & -1.63 & 0.10 \\
\hline
\end{tabular}

${ }^{a}$ The change over time in Suicidal Thinking (Items 5, 6 and 7) on the Concise Health Risk Tracking Scale-Self Report 7-Item (CHRT-SR (Tas compared between the two groups (Immediate Thrive Intervention, INT vs Waitlist Control, WLC) using a logistic regression model via a Generalized Estimating Equation analysis of repeated measures while adjusting for the baseline measure of suicidal thinking. The probabilities were modeled as agreement (presence) with suicidal thinking (yes/no)

${ }^{b}$ Estimated Odds Ratio $(\mathrm{OR})<1$ indicated lower predicted odds of suicidal ideation for INT vs WLC

'Odds Ratio 95\% Confidence Interval (Cl) with corresponding Z-statistic and p-value

${ }^{\mathrm{d}}$ Overall difference in odds (baseline to week 8) between the INT AND WLC groups

$16.5 \%$ achieved remission by week 8 (defined as PHQ-9 scores $<5$.) Of those with moderate or greater symptom severity at baseline (PHQ-9 of $\geq 10)$ and follow-up data available $(n=51), 15.7 \%$ achieved remission by week 8 . No participants that achieved remission at week 4 subsequently relapsed (PHQ-9 $\geq 10$ ) at week 8 . Only $22.8 \%$ reported experiencing suicidal ideation (PHQ-9 Item 9 scores of 1,2 or $3, n=79$ ) at week 8 compared to the baseline proportion of $44.5 \%$.

The mean number of lessons completed was $4.6( \pm 5.7)$. A majority $(69 \%)$ of INT group participants completed between 3 and 9 lessons (out of 30 total lessons). The cumulative number of lessons completed was significantly associated with decreased PHQ-9 $[F(1,371.4)=4.97, p=0.026]$ and GAD-7 $[F(1,369.5)=13.8, p<0.001]$ scores. Lesson completion did not 
Table 3 Longitudinal outcomes of secondary clinical measures for intent-to-treat population

\begin{tabular}{|c|c|c|c|c|c|c|c|c|c|c|}
\hline \multirow[t]{2}{*}{ Secondary outcome measures } & \multicolumn{2}{|c|}{ Baseline $^{a}$} & \multicolumn{3}{|l|}{ Week $8^{b}$} & \multicolumn{2}{|l|}{ Slope ${ }^{c}$} & \multicolumn{2}{|l|}{ Effect $^{d}$} & \multirow{2}{*}{$\begin{array}{l}\text { Effect size } \\
\text { Cohen d }\end{array}$} \\
\hline & Mean & SD & LS mean & (SE) & $95 \% \mathrm{Cl}$ & Mean & $(\mathrm{SE})$ & F-statistic (df) & p-value & \\
\hline \multicolumn{11}{|l|}{ Depression symptom severity ${ }^{f}$} \\
\hline WLC group $(n=230)$ & 13.8 & $(5.5)$ & 10.6 & $(0.29)$ & {$[10.0,11.2]$} & -3.0 & $(0.34)$ & $F(1,899.9)=7.24$ & 0.007 & 0.08 \\
\hline INT group $(n=218)$ & 13.7 & $(5.3)$ & 9.3 & $(0.39)$ & {$[8.56,10.1]$} & -4.3 & $(0.54)$ & & & \\
\hline \multicolumn{11}{|l|}{ Anxiety symptom severity ${ }^{g}$} \\
\hline WLC group $(n=230)$ & 12.5 & $(5.0)$ & 9.9 & $(0.27)$ & {$[9.37,10.4]$} & -2.6 & $(0.32)$ & $F(1,899.9)=2.98$ & 0.085 & 0.10 \\
\hline INT group $(n=218)$ & 12.5 & $(4.8)$ & 9.1 & $(0.36)$ & {$[8.42,9.82]$} & -3.3 & $(0.51)$ & & & \\
\hline \multicolumn{11}{|l|}{ Work and social functioning ${ }^{h}$} \\
\hline WLC group $(n=229)$ & 22.4 & $(8.5)$ & 19.5 & $(0.42)$ & {$[18.7,20.4]$} & -2.4 & $(0.49)$ & $F(1,895)=0.48$ & 0.491 & 0.06 \\
\hline INT group $(n=215)$ & 21.8 & (7.4) & 19.1 & $(0.56)$ & {$[18.0,20.2]$} & -2.8 & $(0.78)$ & & & \\
\hline \multicolumn{11}{|l|}{ Resilience $^{i}$} \\
\hline WLC group $(n=229)$ & 20.9 & $(6.2)$ & 22.1 & $(0.27)$ & {$[21.6,22.7]$} & 1.3 & $(0.32)$ & $F(1,895)=0.44$ & 0.510 & 0.13 \\
\hline INT group $(n=215)$ & 20.9 & $(6.2)$ & 21.8 & $(0.37)$ & {$[21.1,22.6]$} & 1.0 & $(0.52)$ & & & \\
\hline
\end{tabular}

WLC waitlist control group; INT immediate Thrive intervention group

a'Week 0 (baseline) mean and standard deviation

${ }^{b}$ Week 8 least square (LS) mean and standard error (SE)

${ }^{c}$ Mean slope and standard error (SE) from baseline to week 8

${ }^{d} p$-value was associated with the test (F-statistic, Type III Tests of Fixed Effects) of the overall time period difference

${ }^{e}$ Cohen $d$ between group effect size for unequal group sizes

${ }^{f}$ Patient Health Questionnaire-9 (PHQ-9) score range $=0-27$

${ }^{9}$ Generalized Anxiety Disorder Scale-7 (GAD-7) score range $=0-21$

hWork and Social Adjustment Scale (WSAS) score range $=0-40$

'Connor-Davidson Resilience Scale (CD-RISC-10) score range $=0-40$

show statistically significant associations with measures of social adjustment $[F(1,369.5)=13.8, p=0.948]$ or resilience $[F(1,355.7)=3.44, p=0.065]$.

\section{Discussion}

The main objective of this study was to replicate and expand on our prior RCT which had shown that a fully automated iCBT program (Thrive) was effective in reducing depressive symptoms (primary outcome) and anxiety symptoms (secondary outcome), and improving social functioning and resilience (secondary outcomes) in a study sample of rural US adults, compared to a waitlist control group. In the present study, we used suicidal thinking as the primary outcome, retaining as a secondary objective a test of Thrive's impact on measures of depression, anxiety, work and social functioning, and resilience. Finally, like in the initial trial, we examined the association of program adherence with each outcome measure. As discussed below, our second trial partially replicated results from our initial trial.

First, the iCBT program did not significantly reduce suicidal thinking (not measured in our initial trial) compared to the WLC group. However, as in the first trial, it resulted in significant reductions of depressive symptoms. We are aware of only three published trials that used self-guided iCBT programs to reduce suicidal ideation which all reported significant intervention effects on suicidal thinking [35-37]. However, these studies are not directly comparable to our study because they specifically recruited adults with suicidal thoughts and used a program ("Living with Suicidal Thoughts") that included additional (to CBT) components specifically designed to target suicidality (Dialectical Behavior Therapy, DBT). Hence, we cannot rule out that our negative findings are a function of sample composition (i.e., our sample did not include enough participants with suicidal thoughts at baseline, thus limiting our ability to detect an intervention effect). For example, a recent meta-analysis found that iCBT effects are stronger for patients with moderate or severe depression compared to less symptomatic patients [38]. Importantly, however, our replication finding of significant improvements in depression symptoms is heartening because depression is common in US 
rural residents and yet they face substantial barriers to mental health care (e.g., dearth of providers, long distances to care providers, financial barriers) [13] that iCBT programs may help to address [17, 39].

We did not find statistically significant group differences in secondary outcomes of anxiety symptom severity, social functioning, or resilience, in contrast to our first trial where small to moderate between-group effect sizes favored the INT group. Of the iCBT trials that have examined anxiety, the results are also mixed with four studies showing no significant group differences [40-43] and three reporting small group differences [44-46]. Four trials assessing daily functioning/disability found either no significant group differences $[44,48]$ or a moderate effect size $[46,49]$. We did not identify any studies examining iCBT impacts on resilience. As has been found in other studies [18, 22, 44], greater program adherence was associated with larger improvements in depression and anxiety symptoms and differences across published results in the secondary outcomes of interest in our trial may in part reflect differences in program adherence. Despite expert agreement that adherence is an important potential predictor of treatment outcome in digital interventions $[18,44]$, there is no agreed-upon measure or set of measures of program adherence in iCBT or other digital mental health interventions. To increase adherence, experts suggest that Design Thinking should be applied when developing future digital mental health interventions [49]. Design Thinking addresses existing barriers to user engagement, which is a primary path to program adherence and promises to overcome the "empathy gap" that may exist with digital mental health programs by focusing on the emotional and motivational nuances of users [49].

Our findings of an iCBT intervention's favorable impact on reducing depression symptoms found here and in previous studies [21, 40-48], along with evidence of cost-effectiveness [39] should encourage policies that support dissemination of these intervention types for enhancing the work of mental health professionals. Rural mental health practitioners and other community leaders should be aware of these programs as complementary to traditional forms of care. Future work is needed on how best to integrate standard clinical care with iCBT intervention delivery, adherence, and progress towards improving mental wellbeing and quality of life. Emerging innovative technologies hold vast potential to provide platforms for integration of the spectrum of patient care. Future research should compare the degree to which clinicians actively monitor and engage patient outcomes with iCBT interventions and ultimate patient improvement.

\subsection{Limitations}

Several limitations should be considered when interpreting our findings. We decided to use the 3-item CHRT measure, which focuses specifically on suicidal ideation; as such, this reductive approach may exclude other complex risk factors for suicide. Moreover, we did not recruit specifically for individuals with suicidal thinking which may have obscured the potential clinical utility of the Thrive program for such individuals. Our 8-week attrition rates were disparate between study groups (nearly $62 \%$ among the INT group and $37 \%$ among the WLC group), which may have skewed our results. The study used self-assessments for each outcome measure, which may underestimate intervention effects compared to clinician-rated assessments [50]. Our adherence analyses were exploratory, as the study design did not systematically vary dosing of the lessons. Last, our findings should not be generalized to clinical populations; this was a pragmatic trial of adults who may or may not have received a mental health diagnosis.

\subsection{Conclusions}

In the context of US rural communities' higher suicide rates and significant barriers to mental health care $[8,13]$, iCBT interventions offer a scalable strategy to deliver CBT skills for reducing depression, a risk factor of suicide ideation and behavior. Increasing evidence supports the effectiveness of iCBT interventions for reducing depression and anxiety [51]. Digital psychiatry interventions in general are showing promise [52]. We expect that with converging trends in technology and digital health science, we will be able to capitalize on innovative ways to address the existing mental healthcare access issues through digital mental health tools. Given the great tragedy of suicide in all communities, further study of the role iCBT programs for reducing depression, anxiety and other suicidal risk factors is clearly warranted.

Acknowledgements This study was funded by the Montana Department of Public Health \& Human Services, House Bill 118 Suicide Prevention Initiative and the Montana Health Care Foundation. We thank Matt Kunz, Director of the Montana Chapter of the National Alliance for Mental Illness, for his leadership and support of the project. 
Disclosure John Greist received royalty payments from Waypoint Health Innovations based on revenue from Thrive use until 2020. The terms of Dr. Greist's financial relationship with Waypoint Health Innovations have been reviewed by Montana State University, and his involvement with this research project has been approved in accordance with its conflict-of-interest policies.

Authors' contributions MS wrote the main manuscript; BM conducted study analyses and prepared tables and figures, and contributed to the writing of methods and results; JG contributed to the writing of the manuscript; RW contributed to the writing of the manuscript. All authors read and approved the final manuscript.

Data availability The data that support the findings of this study are available from the corresponding author upon request.

\section{Declarations}

Competing interests The authors declare no competing interests.

Open Access This article is licensed under a Creative Commons Attribution 4.0 International License, which permits use, sharing, adaptation, distribution and reproduction in any medium or format, as long as you give appropriate credit to the original author(s) and the source, provide a link to the Creative Commons licence, and indicate if changes were made. The images or other third party material in this article are included in the article's Creative Commons licence, unless indicated otherwise in a credit line to the material. If material is not included in the article's Creative Commons licence and your intended use is not permitted by statutory regulation or exceeds the permitted use, you will need to obtain permission directly from the copyright holder. To view a copy of this licence, visit http://creativecommons.org/licenses/by/4.0/.

\section{References}

1. Hirsch JK. A review of the literature on rural suicide: risk and protective factors, incidence, and prevention. Crisis. 2006;27(4):189-99. https://doi.org/10.1037/rmh0000018.

2. Searles VB, et al. Suicides in urban and rural counties in the United States, 2006-2008. Crisis. 2014;35(1):18-26. https://doi.org/10.1027/ 0227-5910/a000224.

3. Ivey-Stephenson AZ, et al. Suicide trends among and within urbanization levels by sex, race/ethnicity, age group, and mechanism of death—United States, 2001-2015. MMWR Surveill Summ. 2017;66(18):1-16. https://doi.org/10.15585/mmwr.ss6618a1.

4. Leavitt RA, et al. Suicides among American Indian/Alaska Natives-National Violent Death Reporting System, 18 States, $2003-2014$. MMWR Morb Mortal Wkly Rep. 2018;67(8):237-42. https://doi.org/10.15585/mmwr.mm6708a1.

5. Steelesmith DL, et al. Contextual factors associated with county-level suicide rates in the United States, 1999 to 2016. JAMA Netw Open. 2019;2(9):e1910936-e1910936. https://doi.org/10.1001/jamanetworkopen.2019.10936.

6. Goldstick JE, Carter PM, Cunningham RM. Current epidemiological trends in firearm mortality in the United States. JAMA Psychiat. 2020. https://doi.org/10.1001/jamapsychiatry.2020.2986.

7. Shiels MS, et al. Trends in mortality from drug poisonings, suicide, and alcohol-induced deaths in the United States from 2000 to 2017. JAMA Netw Open. 2020;3(9):e2016217-e2016217. https://doi.org/10.1001/jamanetworkopen.2020.16217.

8. Pettrone K, Curtin S. NCHS data brief: urban-rural differences in suicide rates, by sex and three leading methods: United States, $2000-2018$. Atlanta: Centers for Disease Control and Prevention; 2020.

9. Hirsch JK, Cukrowicz KC. Suicide in rural areas: an updated review of the literature. J Rural Mental Health. 2014;38(2):65. https://doi.org/ $10.1037 / \mathrm{rmh} 0000018$.

10. Steelesmith $\mathrm{DL}$, et al. Contextual factors associated with county-level suicide rates in the United States, 1999 to 2016. JAMA Open. 2019;2(9): e1910936. https://doi.org/10.1001/jamanetworkopen.2019.10936.

11. Smith NDL, Kawachi I. State-level social capital and suicide mortality in the 50 US states. Soc Sci Med. 2014;120:269-77. https://doi.org/ 10.1016/j.socscimed.2014.09.007.

12. Summers-Gabr NM. Rural-urban mental health disparities in the United States during COVID-19. Psychol Trauma US. 2020;12(S1):S222-4. https://doi.org/10.1037/tra0000871.

13. Schure MB, et al. Thrive for Montana: final report. Bozeman: Montana State University; 2020.

14. Centers for Disease Control and Prevention. BRFSS prevalence \& trends data. Atlanta: Centers for Disease Control and Prevention; 2019.

15. Centers for Disease Control and Prevention. Suicide mortality by state. 2019. https://www.cdc.gov/nchs/pressroom/sosmap/suicide-morta lity/suicide.htm. Accessed 4 Nov 2020.

16. Monteith LL, et al. Preventing suicide in rural communities during the COVID-19 pandemic. J Rural Health. 2020. https://doi.org/10.1111/ jrh.12448.

17. Vallury KD, Jones M, Oosterbroek C. Computerized cognitive behavior therapy for anxiety and depression in rural areas: a systematic review. J Med Internet Res. 2015;17:e139. https://doi.org/10.2196/jmir.4145.

18. Wilhelm S, et al. Cognitive-behavioral therapy in the digital age: presidential address. Behav Ther. 2020;51(1):1-14. https://doi.org/10. 1016/j.beth.2019.08.001.

19. Arnberg FK, et al. Internet-delivered psychological treatments for mood and anxiety disorders: a systematic review of their efficacy, safety, and cost-effectiveness. PLoS ONE. 2014;9(5): e98118. https://doi.org/10.1371/journal.pone.0098118.

20. Schure $M B$, et al. Exploring perceptions of a computerized cognitive behavior therapy program in a US rural western state. J Rural Mental Health. 2018;42(3-4):174-83. https://doi.org/10.1037/rmh0000102.

21. Schure MB, et al. Use of a fully automated Internet-based cognitive behavior therapy intervention in a community population of adults with depression symptoms: randomized controlled trial. J Med Internet Res. 2019;21(11): e14754. https://doi.org/10.2196/14754. 
22. Schure $M$, et al. Twelve-month follow-up to a fully automated Internet-based cognitive behavior therapy intervention for rural adults with depression symptoms: single-arm longitudinal study. J Med Internet Res. 2020;22(10): e21336. https://doi.org/10.2196/21336.

23. Kroenke K, Spitzer RL, Williams JB. The PHQ-9: validity of a brief depression severity measure. J Gen Intern Med. 2001;16(9):606-13. https:// doi.org/10.1046/j.1525-1497.2001.016009606.x.

24. Waypoint Health Innovations. Waypoint Health Innovations. 2021. https://waypointhealth.com/thrive/. Accessed 15 Apr 2020.

25. Ostacher MJ, et al. A clinical measure of suicidal ideation, suicidal behavior, and associated symptoms in bipolar disorder: Psychometric properties of the Concise Health Risk Tracking Self-Report (CHRT-SR). J Psychiat Res. 2015;71:126-33. https://doi.org/10.1016/j.jpsychires. 2015.10.004.

26. Trivedi MH, Wisniewski SR, Morris DW. Concise Health Risk Tracking Scale: a brief self-report and clinician rating of suicide risk. J Clin Psychiat. 2011;72(6):757-64. https://doi.org/10.4088/JCP.11m06837.

27. Löwe B, et al. Validation and standardization of the Generalized Anxiety Disorder Screener (GAD-7) in the general population. Med Care. 2008;46(3):266-74. https://doi.org/10.1097/MLR.0b013e318160d093.

28. Spitzer RL, et al. A brief measure for assessing generalized anxiety disorder: the GAD-7. Arch Intern Med. 2006;166(10):1092-7. https:// doi.org/10.1001/archinte.166.10.1092.

29. Mundt JC, et al. The Work and Social Adjustment Scale: a simple measure of impairment in functioning. Brit J Psychiat. 2002;180(5):461-4. https://doi.org/10.1192/bjp.180.5.461.

30. Campbell-Sills L, Stein MB. Psychometric analysis and refinement of the Connor-Davidson Resilience Scale (CD-RISC): validation of a 10-item measure of resilience. J Trauma Stress. 2007;20(6):1019-28. https://doi.org/10.1002/jts.20271.

31. Lipsitz SR, Kim K, Zhao L. Analysis of repeated categorical data using generalized estimating equations. Stat Med. 1994;13(11):1149-63. https://doi.org/10.1002/sim.4780131106.

32. Little RJ. Modeling the drop-out mechanism in repeated-measures studies. J Am Stat Assoc. 1995;90(431):1112-21. https://doi.org/10. 1080/01621459.1995.10476615.

33. Rubin DB. Inference and missing data. Biometrika. 1976;63(3):581-92. https://doi.org/10.1093/biomet/63.3.581.

34. Wolfinger R, Chang M. Comparing the SAS GLM and MIXED procedures for repeated measures. In: Proceedings of the Twentieth Annual SAS Users Group Conference. Cary: SAS Institute; 1998.

35. van Spijker BA, van Straten A, Kerkhof AJFM. Effectiveness of online self-help for suicidal thoughts: results of a randomised controlled trial. PLoS ONE. 2014;9(2): e90118. https://doi.org/10.1371/journal.pone.0090118.

36. van Spijker BA, et al. Effectiveness of a web-based self-help program for suicidal thinking in an Australian community sample: randomized controlled trial. J Med Internet Res. 2018;20(2): e15. https://doi.org/10.2196/jmir.8595.

37. De Jaegere $\mathrm{E}$, et al. The online treatment of suicidal ideation: a randomised controlled trial of an unguided web-based intervention. Behav Res Ther. 2019;119: 103406. https://doi.org/10.1016/j.brat.2019.05.003.

38. Karyotaki E, et al. Internet-based cognitive behavioral therapy for depression: a systematic review and individual patient data network meta-analysis. JAMA Psychiat. 2021;78(4):361-71. https://doi.org/10.1001/jamapsychiatry.2020.4364.

39. Thase ME, et al. Improving cost-effectiveness and access to cognitive behavior therapy for depression: providing remote-ready, computerassisted psychotherapy in times of crisis and beyond. Psychother Psychosom. 2020. https://doi.org/10.1159/000508143.

40. Terides MD, et al. Increased skills usage statistically mediates symptom reduction in self-guided internet-delivered cognitive-behavioural therapy for depression and anxiety: a randomised controlled trial. Cog Behav Ther. 2018;47(1):43-61. https://doi.org/10.1080/16506073. 2017.1347195.

41. Berger T, et al. Evaluating an e-mental health program ("deprexis") as adjunctive treatment tool in psychotherapy for depression: results of a pragmatic randomized controlled trial. J Affect Disord. 2018;227:455-62. https://doi.org/10.1016/j.jad.2017.11.021.

42. Meyer B, et al. Effects of an Internet intervention (Deprexis) on severe depression symptoms: randomized controlled trial. Internet Interv. 2015;2(1):48-59. https://doi.org/10.1016/j.invent.2014.12.003.

43. Phillips R, et al. Randomized controlled trial of computerized cognitive behavioural therapy for depressive symptoms: effectiveness and costs of a workplace intervention. Psychol Med. 2014;44(4):741-52. https://doi.org/10.1017/S0033291713001323.

44. Beevers CG, et al. Effectiveness of an internet intervention (Deprexis) for depression in a United States adult sample: a parallel-group pragmatic randomized controlled trial. J Consult Clin Psychol. 2017;85(4):367-80. https://doi.org/10.1037/ccp0000171.

45. Mira A, et al. An Internet-based program for depressive symptoms using human and automated support: a randomized controlled trial. Neuropsych Dis Treat. 2017;13:987-1006. https://doi.org/10.2147/NDT.S130994.

46. Kleiboer A, et al. A randomized controlled trial on the role of support in Internet-based problem solving therapy for depression and anxiety. Behav Res Ther. 2015;72:63-71. https://doi.org/10.1016/j.brat.2015.06.013.

47. Arean PA, et al. The use and effectiveness of mobile apps for depression: results from a fully remote clinical trial. J Med Internet Res. 2016;18(12): e330. https://doi.org/10.2196/jmir.6482.

48. Meyer B, et al. Effectiveness of a novel integrative online treatment for depression (Deprexis): randomized controlled trial. J Med Internet Res. 2009;11(2): e15. https://doi.org/10.2196/jmir.1151.

49. Scholten $\mathrm{H}$, Granic I. Use of the principles of design thinking to address limitations of digital mental health interventions for youth. J Med Internet Res. 2019;21(1): e11528. https://doi.org/10.2196/11528.

50. Cuijpers $\mathrm{P}$, et al. Self-reported versus clinician-rated symptoms of depression as outcome measures in psychotherapy research on depression: a meta-analysis. Clin Psych Rev. 2010;30(6):768-78. https://doi.org/10.1016/j.cpr.2010.06.001.

51. Andrews $\mathrm{G}$, et al. Computer therapy for the anxiety and depression disorders is effective, acceptable and practical health care: an updated meta-analysis. J Anxiety Disord. 2018;55:70-8. https://doi.org/10.1016/j.janxdis.2018.01.001.

52. Torous J, et al. The growing field of digital psychiatry: current evidence and the future of apps, social media, chatbots, and virtual reality. World Psychiat. 2021;20(3):318-33. https://doi.org/10.1002/wps.20883.

Publisher's Note Springer Nature remains neutral with regard to jurisdictional claims in published maps and institutional affiliations. 